\title{
Laws of Nature and Counterparts ${ }^{1}$
}

\section{Esteban Céspedes}

\begin{abstract}
The events of nature are, at first glance, related to each other in a necessary way, as if they were subject to certain rules, a fact that is closely linked with the ontology of natural laws. However, there are several conflicting theories about their existence, such as the Humean view as well as realism. Mumford proposes a third way: to accept natural regularity, but deny that this is due to the real existence of natural laws. Finally, some ideas about a possible combination between nomological realism and Lewis's counterpart theory will be considered in order to improve the first. I will defend realism about regularities as well as about laws and show that such a point of view is not only compatible with counterparts, but that it could be supported by them.
\end{abstract}

Key words: Natural law, nomological realism, modality, supervenience

\section{Regularity without Laws}

$\mathrm{T}$

he realist conception of natural laws establishes that these are real entities in a strict metaphysical sense. They do not supervene on other most basic entities, nor are they apparent: they are real and determine the structure of the natural world. Some of the important detailed views on realism are presented by Tooley, who argues that the truth-makers of laws must be universals, ${ }^{2}$ and Woodward, whose view consists in founding laws on causal connections between particular objects. ${ }^{3}$ According to the general realist account, natural events are connected by regularities and can be predicted by the conjunction of natural laws and particular facts. This view, also called nomological realism, which according to Mumford has two basic premises. ${ }^{4}$ The first is that the world has certain features, such as regularity, universality, objectivity, natural necessity, and so on. The second premise is that these features depend on the real ontological existence of natural laws. One may deny that these features exist, but it cannot be denied that laws exist. With regard to the connection between prediction and natural laws, the Hempel's interpretation of explanation and prediction is quite orientating.

${ }^{1}$ I am thankful to José Tomás Alvarado for valuable comments. This article was supported by CONICYT and by the Konrad Adenauer Foundation.

2 M. Tooley, "The Nature of Laws," in Canadian Journal of Philosophy, 7:4 (1977).

3 Woodward, J., "Realism about Laws," in Erkenntnis, 36:2 (1992).

${ }^{4}$ S. Mumford, "Laws and Lawlessness," in Synthese, 144:3 (2005), 403. 
According to Hempel \& Oppenheim,,$^{5}$ the explanation of an event must have the following elements: (1) a set of propositions that assert the occurrence of certain events or antecedent conditions, and (2) a set of universal hypotheses or general laws. The latter have the form of universal natural laws and, in conjunction with a given set of propositions defined by (1), other events can be deduced by those laws. Without them there is no prediction and no scientific knowledge, therefore, natural laws are real entities.

Here is the opposing view. According to Hume, events of nature are entirely separated, and if an event occurs after another, it is not possible to perceive any real connection between them. However, as our ideas come from impressions of sense and we have no impressions of those connections, it is meaningless to talk about them, either in the context of philosophical reasoning or in everyday context. This view is called Humean lawlessness and says that connections between events are not intrinsic to nature, but that we understand them in the form of habits. Talking about any kind of connection between events such as natural or causal laws is completely meaningless.

The best formulation of the Humean conception of natural laws is the theory of Ramsey-Lewis, whose foundations are quite obvious, according to Mumford. The problem of Humean supervenience, the metaphysical basis of this theory, may be presented as follows. According to Lewis, ${ }^{6}$ the world is made, in part, by natural laws, probability and causal relations and consists of a distribution of space-time points of local qualitative character. ${ }^{7}$ Can two possible worlds differ from each other with regard to its laws without differing with respect to its local character? As argued by Armstrong, ${ }^{8}$ Humean supervenience claims that natural laws and causes supervene on states of local and particular things. This means, on the basis of local supervenience, that there cannot be any difference between the natural laws of two possible worlds, without a difference between their particular facts. According to Lewis, the supervenience of natural laws (SNL) should be read as follows: ${ }^{9}$

5 C. Hempel \& P. Oppenheim, "Studies in the Logic of Explanation," in Philosophy of Science, 15:2 (1948), 136. See also C. Hempel, Aspects of Scientific Explanation and Other Essays in the Philosophy of Science (New York: Free Press, 1965).

${ }^{6}$ D. Lewis, On the Plurality of Worlds (Oxford: Blackwell, 1986), 14.

${ }^{7}$ Naturally, Lewis also accepts Ramsey's influence in his notion of natural laws: 'Few would deny that laws of nature, whatever else they may be, are at least exceptionless regularities. See D. Lewis, Philosophical Papers Volume II (Oxford: Oxford University Press, 1986), xi. Not all regularities are laws, of course. But, following the lead of (a short temporal segment of) Ramsey, I suggest that the laws are the ones that buy into those systems of truths that achieve an unexcelled combination of simplicity and strength. See also F. Ramsey, "Universals of Law and of Facts," in Foundations: Essays in philosophy, Logic, Mathematics and Economics (London: Routledge \& Kegan Paul. 1978).

${ }^{8} \mathrm{D}$. Armstrong, "Going through the open door again: counterfactual versus singularist theories of causation," in Reality and Human Supervenience: Essays on the Philosophy of David Lewis (New York: Rowman \& Littlefield, 2001), 164.

${ }^{9}$ Lewis, On the Plurality of Worlds, 16. 
SNL: No two worlds differ in their laws without differing in its distribution of local character and quality.

$$
\text { SNL: } \neg\left[\left(\mathrm{w}^{1} \mathrm{~L} \text {-differs } \mathrm{w}^{2}\right) \& \neg\left(\mathrm{w}_{1} \mathrm{C} \text {-differs } \mathrm{w}_{2}\right)\right]
$$

The relation of supervenience may have the meaning of either reduction, ${ }^{10}$ explanation or even implication. But the goal here is not to make an analysis of the supervenience relation. The fact is that supervenience shows a particular type of connection between two states, where one of them is more fundamental than the other and whose changes determine changes in the facts that supervene on them. ${ }^{11}$ For this reason, according to Mumford, the concept of 'law' deserves hardly to be named like this in a Humean sense, where natural laws supervene on a four-dimensional physicalist basis. Mumford describes this Humean basis in five aspects: ${ }^{12}$ there are four-dimensional events, these events are fundamental, scientific theories would explain them, everything supervenes on them, and they are modally unconnected. The aspect of the empirical explainability of these events seems to be especially problematic. But that is an issue against Humean lawlessness that I am going to consider in the last sections of this paper.

Because they are the foundation of everything that happens in nature, these events just happen: they are not governed by natural laws, since these are products of supervenience. Only if one takes the concept of natural law in the strict sense, i.e. as a real entity that determines the structure of the world and its events, then it can be said that the Humean lawlessness does not admit natural laws. It does not agree with any of the two premises of nomological realism, while Mumford's realist lawlessness accepts the first condition (that regularities exist) but not the second. ${ }^{13}$ This means that the world actually has the features mentioned above, but this regularity is not due to the real existence of laws of nature.

Another main difference between the realist lawlessness and the other two opposing theories is that neither of both considers the modal connections as foundation of the dynamism between events. The nomological realist uses the notion of 'law' in order to explain the dynamism of nature, while Humean lawlessness excludes modality in one of its premises. Modal aspects of reality may account for this dynamism perfectly if seriously. According to Mumford, natural laws (or regular relationships between physical events) should be understood from their modal character, i.e. inside the modal logic space. ${ }^{14}$ The interesting aspect in thinking of natural laws as contingent is that it enables the notion of discovery.

\footnotetext{
10 See J. Kim, "Postscript on Supervenience," in Supervenience and Mind: Selected Philosophical Essays (Cambridge: Cambridge University Press, 1993).

11 There are differences between the notions of 'event' and 'fact' that will not be considered in the present work.

12 Mumford, op. cit., 402.

13 Ibid., 406.

${ }^{14}$ For a discussion about the ontology of modal logic space, see J. T., Alvarado, “¿Qué es el espacio ontológico modal?," in Philosophica, 29 (2006), 7-44.
} 
However, if it is absurd within realist lawlessness to ignore the contingency of laws (believing that a law is by definition always necessary), it might be also absurd to establish that all natural laws are contingent. The reason for this (returning to the previous example) is that the property of being a photon may have different causal roles in possible worlds where the common physical laws were different. ${ }^{15}$ The same photon may sometimes produce some effects at long distances very easily, and at other times, under the same conditions, it probably won't have the same capacity. The counterparts of a single photon in the actual world could have a speed $c$ in the possible world $\mathrm{w}_{1}$, the speed of $2 c$ at $\mathrm{w}_{2}$ or an always varying speed at w3. This would mean a counterexample to one essential property $(\phi)$ as defined on counterpart theory by Lewis. ${ }^{16}$ Let the first Greek letters represent entities and ' $\mathrm{I}$ ' be the binary relation that associates an entity with the world in which it exists. Let also ' $\mathrm{W}$ ' be the predicate of being a world and ' $\mathrm{C}$ ' the binary relation from the set of entities to the set of its counterparts: ${ }^{17}$

$$
\exists \alpha\left\{\left[a @ \& \forall \delta\left[I \delta @ \rightarrow\left(\psi^{\varrho} \delta \leftrightarrow \delta=\alpha\right)\right] \& \exists \beta \exists \gamma\left(\mathrm{W} \beta \& I \gamma \beta \& C \gamma \alpha \& \neg \phi^{\beta} \gamma\right)\right\}\right.
$$

I will explain that example later with more detail. For Mumford, the contingency of laws is senseless and that is a perfect result in favour of realist lawlessness, since natural laws are not entities at all. They are not real, as essential or intrinsic properties are, and they are neither necessary nor contingent. The only things in nature are the regular features and relations, such as universality, natural necessity and objectivity.

\section{Necessity, Sentences, and Properties}

In order to explain the necessity of natural laws and scientific research results, Brian Ellis draws the distinction between the necessary propositions de re and the necessary propositions de dicto. ${ }^{18}$ This should be related to the difference between nominal essences and real essences. The nominal essence of something is not necessarily a natural class and is defined as a set of properties which are classified inside a language. The real essence, on the other side, is independent of language, can be discovered empirically and is what defines the kind of things, which is the essence of.

The distinction between de dicto necessity and de re necessity lies, however, in the fact that the former corresponds to the modal assignment of a proposition, not to the property of a thing, which is the case for the latter. Thus, the proposition that expresses the necessity between an entity $x$ and the property of being, e.g. a photon can be analysed as follows:

\footnotetext{
15 Mumford, op. cit., 407.

${ }^{16}$ Lewis, "Counterpart Theory and Quantified Modal Logic."

17 Counterparts are an alternative to the identity between objects in different possible worlds, so that the proposition 'It is possible that $\mathrm{x}$ is $\mathrm{P}$ ' has not the meaning of 'There is a possible world where $\mathrm{x}$ is $\mathrm{P}$ ', but of 'There is a possible world where a counterpart of $\mathrm{x}$ is P.'

${ }^{18}$ B. Ellis, Scientific Essentialism (Cambridge: Cambridge University Press, 2001), 32.
} 
a) De dicto: Necessarily there is an $\mathrm{x}$ such that $\mathrm{x}$ is a photon.

\section{$\exists \mathrm{x}(\varphi \mathrm{x})$}

b) De re: There is an $\mathrm{x}$ such that $\mathrm{x}$ is necessarily a photon.

\section{$\exists \mathrm{x} \square(\varphi \mathrm{x})$}

This presents inevitably the question about natural laws and what should be their modal interpretation, for it is known that natural laws must be necessary in some sense. The problem is in what sense. Ellis argues that both de dicto necessity and de re necessity are true in all possible worlds, because their distinction lies in their natural and real basis, rather than their modal character. ${ }^{19}$ The first is based on the conventions of language, while the second does not. Natural laws correspond to a metaphysical necessity, and every proposition that is necessary for reasons other than metaphysical, may be either de dicto or de re. A de dicto necessity, for instance, 'Every triangle has three corners' is true by definition corresponding to language and not because the natural class of 'triangle' is such that its members have three corners.

To reject scientific conventionalism, whose supporters assert that there are neither metaphysical necessities nor de re necessities (only de dicto), Ellis criticises the Humean conception of natural laws, because natural necessity cannot be a simple generalisation. ${ }^{20}$ The natural and metaphysical necessity of laws takes Ellis to claim a scientific essentialism; according to which causal connections and propensities of things are real and intrinsic properties of nature, e.g., that electrons have a negative charge. Scientific essentialism is also realistic about entities that are part of the constitution of nature, like the existence of electrons themselves.

According to Ellis, natural laws are strictly necessary in the sense that they are descriptions that could not have been otherwise. Therefore, there is no place in his theory for contingent laws. This is a problem for Mumford, because to put aside the contingency of natural laws (if they were real entities) means also to put aside their metaphysical reason. ${ }^{21}$ Some definitions of de $r e$ and de dicto interpretations of necessity according to Lewis are going to be briefly explained in the following section, in order to make clear this problem.

\section{Counterpart Theory}

Essentialism (including Ellis's scientific essentialism), under which entities have some properties necessarily and others contingently, can be interpreted in two ways, depending on whether the propositions are analysed as de dicto necessities or as de re necessities. There are accounts about laws of nature that

19 Ibid., 33.

${ }^{20}$ Ibid., 43.

${ }^{21}$ The definition of 'metaphysical reason' is a topic by itself and I am not going to explain it here in detail. However, it can be argued that the cause of an entity might be the best candidate if one is looking for its metaphysical reason. 
consider many intervening grades of modality, in order to solve these problems. Koslow establishes that a specific physical law narrows the members of a set of possibilities in such a way, that they can fail to have some certain functional property. ${ }^{22}$ According to Lewis's counterpart theory, the fact that an attribute $\varphi$ corresponds necessarily to a singular term $\zeta$ is the same as saying that the attribute is essential to the object denoted by this term. ${ }^{23}$ De dicto interpretations are expressed as follows:

$$
\forall \beta\left(\mathrm{W} \beta \rightarrow \exists \alpha\left(\operatorname{I} \alpha \beta \& \forall \delta\left(\operatorname{I} \delta \beta \rightarrow\left(\psi^{\beta} \delta \leftrightarrow \delta=\alpha\right) \& \psi^{\beta} \alpha\right)\right)\right)
$$

Another reading of this formula should be: 'For all $\beta$, if $\beta$ is a possible world, there exists a unique object which has the property $\varphi$.' A de re interpretation, on the other hand, is made by singling out an individual in the actual world (@) and then by attaching that property to all its counterparts:

$$
\exists \alpha\left(I \alpha @ \& \forall \delta\left(I \delta @ \rightarrow\left(\psi^{\circledR} \delta \leftrightarrow \delta=\alpha\right)\right)\right) \& \forall \beta \forall \gamma\left((\mathrm{W} \beta \& I \gamma \alpha \& C \gamma \alpha) \rightarrow \psi^{\circledR} \gamma\right)
$$

This formula states that the actual world has a single object, such that for every counterpart $\gamma$ of that object in any possible world $\beta, \gamma$ has the property $\varphi$. In other words, a de re interpretation means that the property of a singular object in the actual world is essential, if and only if, every counterpart of that object instantiates the same property. This is the definition to be adopted when we are confronted with an analysis of essential features. The necessary fact, e.g. that $x$ has the speed $c$, refers to the property of having the velocity $c$ under certain conditions, an essential property of a given particle. That does not mean that the proposition ' $\mathrm{x}$ has the speed $c$ ' is true in all possible worlds. Emphasis should therefore be put on the property of that object rather than on the proposition that describes it. Stated in a language foreign to counterpart theory, the fact that having the speed $c$ under certain conditions is an essential property of this particle means that every time this particle is involved under certain conditions, it will travel at the speed of c. Clearly, the notion of essential property can be used as a way to analyse natural laws.

\section{Counterparts and Realism}

In this section it will be argued that counterpart theory might be an alternative to the problems raised by the different conceptions of the ontology of natural laws. It should be noted, however, that to hold a modal theory supported by Lewis does neither mean agreeing with Humean lawlessness nor with the Ramsey-Lewis account. Although the notion of natural law in general can be

\footnotetext{
22 A. Koslow, "Laws and Possibilities," in Philosophy of Science, 71:5 (2004).

${ }^{23}$ Lewis, "Counterpart Theory and Quantified Modal Logic," 120.
} 
analysed including all different sciences, I prefer to have in mind only an example coming from physics, i.e. the example of photons in vacuo. It could be criticised, on one side, either that an account about scientific laws must include all of them or, on the other side, that it should focus only on a specific area of science. For the present purposes I will stay only in physical examples, in order to satisfy the second complaint, and leave open the ideas for other branches of science, in order to satisfy the first. I think that laws are real and would like to suggest that using counterparts does not only improve the way in which laws are expressed, but also gives good reasons to believe that they exist. Counterpart theory is not incompatible with realism about laws. I admit it is hard to find a positive argument for nomological realism inside counterpart theory, but at least it is possible to consider them together. Besides, if a law about certain physical entity is expressed in terms of counterparts, we can always find a counterpart of that entity. In a certain way, the law is the condition for the natural features it describes. It might be argued that counterpart theory does not give an alternative to modal logic, but that it rather avoids modal logic. Perhaps it is right to say that it is not an alternative. I would prefer to think that in some cases it does not matter if one adopts one position or the other. Nevertheless, in order to solve certain problems, a point of view related to laws of nature must tend to prefer counterparts.

In addition to what was mentioned above, according to Lewis, natural laws are propositions which, in implication with other propositions, become counterfactual conditionals (such as 'If this entity was traveling in the vacuo, it would have a speed c). ${ }^{24}$ If from the antecedent of a counterfactual conditional, combined with a set of general laws logically consistent, we obtain the consequent of such counterfactual, then the conditional is true. But the notion of natural law would not be something stable then, it would rather depend on the conditional. When the conditional 'If this entity was traveling in vacuo, it would have a speed $c$ ' is true in a possible world $\mathrm{w}_{1}$, which is within the worlds which are governed by some law of nature L, but turns out to be false in $\mathrm{w}_{2}$, then it must be assumed that $\mathrm{w}_{2}$ does not belong to the set of propositions that make $\mathrm{L}$ true. If there is something called "natural law", then it is only an aspect that supervenes on singled events (four-dimensional events, counterparts, an object's singled instants in the actual world, etc.). Hence this view is considered a form of Humean lawlessness. Given the highly contingent nature of this position, contingent on the fact that there is a point where the counterparts of a photon in particular would no longer have a speed $c$ and would behave otherwise (would play a causal role completely different), an alternative is to say, standing on the side of nomological realism, that natural laws are intrinsic properties of objects. The problem with this, although it is a solution to the contingency of natural laws, is that it implies a strict necessity of them, giving away the modality of natural laws.

It seems perfectly rational to say that there are regular features in the world and that such laws are also governing nature. The laws can be understood as essential properties of objects. However, de re interpretations do not need to rule on the same object in different possible worlds. They can be essential

${ }^{24}$ D. Lewis, Counterfactuals (Oxford: Blackwell, 1973), 72. 
properties of an object's counterparts. A given law L is not necessary because it governs all objects in every possible world; it is necessary for being an essential property of certain objects (or a set of objects). With regard to the theory of Ramsey-Lewis, it is nonsense to think that the regularity of nature rests solely on points in space, for it would be like saying that only the elementary particles are guided by natural laws and that, therefore, the only natural laws that are valid are the laws of subatomic physics. In addition, the postulate that establishes that science can explain and describe all special events is far from consistency, since an adequate scientific explanation needs general laws, and to explain the subvenient elements through natural laws would include the explanandum in the explanans. That is an extremely weak position, unless we think that scientific knowledge is only about descriptions, which is not the case. On the other hand, according to nomological realism of intrinsic properties, as pointed out by Mumford, natural kinds and essential properties end up being considered fundamental and as a result, you do not need the concept of natural law. ${ }^{25}$ But this is not a problem if we consider counterpart theory, where a natural law could be formulated by the following example:

a) Having a speed $c$ under certain conditions is an essential property of the natural kind 'photon' in the actual world and expresses the natural law 'Any counterpart of a photon travels with a speed $c$.'

But this example is also valid if we replace, in the formulation of natural law, the natural kind with that object's counterpart. Thus a natural law may be expressed simply as 'Every photon has a speed $c$.' The interesting problem is that natural laws also govern on individual objects, so that our example can be formulated as follows:

b) There is a single object $a$ in @, such that for every counterpart $g$ of $a, g$ has the property of having the speed $c$ in vacuo and of being a photon.

$$
\begin{aligned}
& \exists \alpha\left\{I \alpha @ \& \forall \delta\left[I \delta @ \rightarrow\left(\psi^{\varrho} \delta \leftrightarrow \delta=\alpha\right)\right] \&\right. \\
& \forall \beta \forall \gamma(W \beta \& I \gamma \beta \& C \gamma \alpha\left.\left.\rightarrow\left(\xi^{\beta} \gamma \& \phi^{\beta} \gamma\right)\right)\right\}
\end{aligned}
$$

Thus, the natural class is modified by an essential property. And that property is not just part of a nominal essence, but of a real essence. Natural laws are in fact expressed by de re necessities with the only difference that they are necessities over counterparts. In this way, laws have not to depend of the descriptions of characteristics of particular entities. However, the fact that all the counterparts of an entity travel at the speed $c$ and are photons does not imply that all photons travel at the speed $c$ in other possible worlds. It might happen that a counterpart $g$ of $a$ is ruled by a law according to which every counterpart of $g$ is a photon but only some of them travel at $c$.

${ }^{25}$ Mumford, op. cit., 406. 


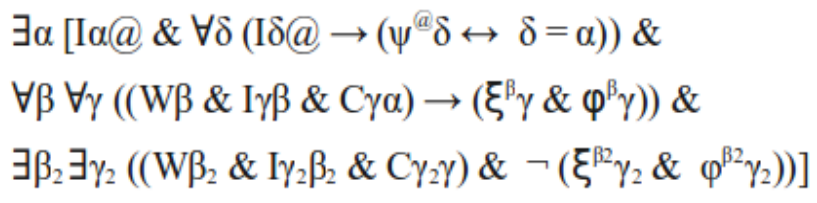

Here is the problem that might arise with regard to realism if applied to singular objects: such an account would have as result (outside counterpart theory) that 'There is an $a$ such that $a$ is a photon and travels at $c$ in all possible worlds', which is not necessarily true. That might be true inside transitive systems of modal logic (like $S 4$ or S5), ${ }^{26}$ but we do not need transitivity to understand scientific knowledge and natural laws. In fact, transitivity could be an obstacle in this domain. If nomological realism is unified with a modal logic without counterparts, then it won't be possible that $a$ was a photon without having the property of travelling at the speed $c$, since this is a natural law that governs the singular object. But in this account, it is perfectly consistent to say that there is a possible world in which there is a counterpart of $a$ photon that is also a photon, but does not travel at $c$, i.e. whose counterparts are all photons but some of them do not travel at $c$.

These considerations try to solve certain problems raised by Mumford on nomological realism and Humean conceptions, while maintaining the real existence of natural laws. Undoubtedly, these thoughts still deserve a much more detailed and careful work than what has been done here. Some important possibly further results of this combination are introduced in the next section.

\section{Prospective Relevance and Conclusions}

This proposal maintains the existence of regularities, universality and necessary connections between events of nature. And not only that: natural laws exist in a metaphysical sense. This position differs in this respect with realist lawlessness and could be significant to analyse notions, on which scientific accounts about reality depend, e.g. randomness and determinism. ${ }^{27}$ It also solves the problem of lack of dynamism of regularities in nature. Natural laws are real (no one says lawlessness), but these are not static propositions without modality, which was the problem of the realist view mentioned by Mumford.$^{28}$ Nomological realists have a demodalised world as a starting condition. In fact, the unification between nomological realism and counterpart theory remains realistic and, of course, modal.

${ }^{26}$ What makes a modal system transitive is the axiom that establishes that 'If a proposition is necessary, then it is necessarily necessary.'

${ }^{27}$ Considerations about natural laws are important in the analysis of deterministic worlds. Alvarado argues that a distinction between deterministic and nondeterministic worlds can only be made by defining natural laws as something more robust than simple Humean regularities. See J. T. Alvarado, "La noción de mundo determinista," in Eikasia, 4:27 (2009).

${ }^{28}$ Mumford, op. cit., 507. 
Furthermore, in this view laws do not refer only to general concepts such as "photon", but also to single entities and their counterparts. One difficulty here is the fifth postulate of counterpart theory, witch states that Nothing is a counterpart of anything else in its world.'29 If we express a law saying that 'All counterparts of this photon travel at speed c', we refer only to those entities that are similar to the photon in other possible worlds, but not to other photons in the actual world. This assumption, quite annoying, could be put aside along with Lewis' modal realism, a topic that does not have to be discussed here.

It is possible to analyse the refutability of theories and natural laws by using counterparts, in the sense that Popper ${ }^{30}$ establishes it: 'There is a counterpart $g$ of $a$, such that $g$ does not travel at a speed c.' This point is quite important to extend the theory of natural laws to the philosophy of scientific knowledge, especially if one considers critical rationalism as a plausible epistemological system. In this direction, this position might be also useful in order to solve the problem of what is (perhaps falsely) called 'inductive inference' by implementing counterparts to the explanation of how theoretical propositions are generalised from particular states of affairs. The propositions of the form 'If every detected $n$ is a photon, then the next detected $n$ will also be a photon' is replaced by 'If $a$ is a photon, then for all $g$, if $g$ is a counterpart of $a$, then $g$ is also a photon.' This formula does not have to become a methodological postulate of inductionism; it is just a form of how an inductive generalisation could look like.

Finally (what does not mean there are no other advantages), a decision theory can be developed by considering counterparts in the laws of nature and Lewis's causal decision theory. ${ }^{31}$ His theory analyses, in an excellent way, some crucial problems of decision making in conjunction with modal logic and counterfactual conditionals. Nevertheless, it leaves some problems unanswered. ${ }^{32}$ Decision theory can be extremely important to understand theory change on the basis of choices made by agents, scientists, or social choices made by scientific groups. ${ }^{33} \mathrm{~A}$ very interesting problem in this field is that scientific decision making could be sometimes closer to paradoxes of rationality than to normal perfect information scenarios.

This paper has presented an analysis of the philosophy of natural laws, by reviewing some important theories about it, including Mumford's realist lawlessness. I considered then the important distinction between de re and de dicto modalities, starting from Ellis's and Lewis's work on this field. In the last sections there are some reflections on the possible unification between nomological

${ }^{29}$ Lewis, "Counterpart Theory and Quantified Modal Logic," 114.

${ }^{30}$ K. Popper, Conjectures and Refutations (London: Routledge \& Kegan Paul, 1963).

${ }^{31}$ D. Lewis, "Causal Decision Theory," in Australasian Journal of Philosophy, 59:1 (1981).

32 To this sort of objections and counterexamples to causal decision theory, see T. Horgan, "Counterfactuals and Newcomb's Problem," in Journal of Philosophy, 78:6 (1981), 331-56.; A. Egan, "Some Counterexamples to Causal Decision Theory," in Philosophical Review, 116:1 (2007), 93-114; P. McKay, "Newcomb's problem: the causalists get rich," in Analysis, 64:2 (2004); E. Céspedes, "La teoría de la decisión de David Lewis y la paradoja de Newcomb," in Limite, 4:20 (2009), 53-68.

33 See W. Harper \& B. Skyrms, Causation in Decision, Belief Change, and Statistics (Dordrecht: Ruwer, 1988). 
realism and counterpart theory. I show that Humean theory of natural laws is circular, if the constituent elements have both to explain nature and to be scientifically explained by general laws (according to the definition of explanation). Moreover, essential properties give a good model on these issues, although there are some weaknesses that could be strengthened by the inclusion of counterpart theory, without giving up the existence of natural laws.

Institut für Philosophie, J. W. Goethe-Universität_Frankfurt am Main, Germany

\section{References}

Alvarado, J. T., “¿Qué es el espacio ontológico modal?,” in Philosophica, 29 (2006), $7-44$. 109.

"La noción de mundo determinista," in Eikasia, 4:27 (2009), 93-

Armstrong, D., "Going Through the Open Door again: counterfactual versus Singularist Theories of causation," in Reality and Humean Supervenience: Essays on the Philosophy of David Lewis, ed. by Preyer and Siebelt (New York: Rowman \& Littlefield, 2001).

Céspedes, E., "La teoría de la decisión de David Lewis y la paradoja de Newcomb," in Limite, 4:20 (2009), 53-68.

Egan, A., "Some Counterexamples to Causal Decision Theory," in Pbilosophical Review, 116:1 (2007), 93-114.

Ellis, B., Scientific Essentialism (Cambridge: Cambridge University Press, 2001).

Harper, W. \& Skyrms, B., Causation in Decision, Belief Change, and Statistics (Dordrecht: Ruwer, 1988).

Hempel, C. \& Oppenheim, P., "Studies in the Logic of Explanation," in Pbilosophy of Science, 15:2 (1948), 135-75. , Aspects of Scientific Explanation and Other Essays in the Philosophy of Science (New York: Free Press, 1965).

Horgan, T., "Counterfactuals and Newcomb's Problem," in Journal of Philosophy, 78:6 (1981), 331-56.

Hume, D., An Enquiry Concerning Human Understanding: A Critical Edition (Oxford: Oxford University Press, 2000).

Kim, J., "Postscript on Supervenience.," in Supervenience and Mind: Selected Philosophical Essays (Cambridge: Cambridge University Press, 1993). Phenomenological Research, 45:2 (1984), 53-76.

Koslow, A., "Laws and Possibilities," in Pbilosophy of Science, 71:5 (2004), 719-729.

Lewis, D. " "Humean Supervenience Debugged," in Mind, 103:412 (1994), 473-90. (1981), 5-30. "Causal Decision Theory," in Australasian Journal of Pbilosophy, 59:1

, "Counterpart Theory and Quantified Modal Logic," in Journal of Philosophy, 65:5 (1968), 113-26. Counterfactuals (Oxford: Blackwell, 1973). , On the Plurality of Worlds (Oxford: Blackwell, 1986). 


\section{NATURE AND COUNTERPARTS}

1986).

Philosophical Papers Volume II (Oxford: Oxford University Press,

Mckay, P., "Newcomb’s problem: the causalists get rich," in Analysis, 64:2 (2004), 187-89.

Mumford, S., "Laws and Lawlessness," in Synthese, 144:3 (2005), 397-413.

Popper, K., Conjectures and Refutations (London: Routledge \& Kegan Paul, 1963).

Ramsey, F., "Universals of Law and of Facts," in Foundations: Essays in philosophy, Logic, Mathematics and Economics (London: Routledge \& Kegan Paul. 1978).

Tooley, M., "The Nature of Laws," in Canadian Journal of Philosophy, 7:4 (1977), 667-98.

Woodward, J., "Realism about Laws," in Erkenntnis, 36:2 (1992), 181-218. 